\title{
EL SISTEMA DE HABILITACIÓN NACIONAL: CRITERIOS Y PROCESO DE EVALUACIÓN
}

\section{Gualberto Buela-Casal}

Facultad de Psicología, Universidad de Granada

\section{RESUMEN}

En este artículo se realiza un detallado análisis del sistema actual de evaluación y selección del profesorado universitario en España. En concreto se analizan los criterios y el proceso de evaluación, la fiabilidad y validez y los concursos de acceso. En primer lugar, se pone de manifiesto que no hay criterios operativos de evaluación predeterminados, dado que estos siempre quedan en manos de los miembros de las comisiones de evaluación, y por ello, ocurre que los criterios varian de una comisión a otra, tal como se demuestra con varios ejemplos de casos reales. En segundo lugar, se demuestra que el proceso de evaluación, aunque está reglamentado en parte, es bastante ambiguo en relación a como aplicar los criterios, pero aun más importante es el problema que se produce por el hecho de que las comisiones puedan actuar hasta con cuatro miembros, es decir, aun faltando tres, dado que se constituye inicialmente con siete; esto es especialmente relevante, pues siempre se mantiene el nivel de obtener al menos cuatro votos para superar una prueba. Lo anterior, junto con el problema de la definición clara de criterios genera en la práctica algunos problemas de fiabilidad y validez. No obstante, se concluye que estas deficiencias son fácilmente subsanables y que por tanto, 
el Sistema de Habilitación Nacional es, sin duda alguna, un sistema mejor que el de la anterior $L R \cup$ y que genera mucho menos endogamia en la selección del profesorado funcionario universitario.

Palabras clave: SELECCIÓN DE PROFESORES, HABILITACIÓN NACIONAL, CONCURSO DE ACCESO

\section{SUMMARY}

This paper presents a detailed analysis of the current system for the assessment and recruitment of university professors in Spain. Specifically, assessment criteria, assessment process, reliability, validity and competitive/entrance procedures are examined. First, it is shown that there are not predefined assessment criteria since these criteria are established by the members belonging to the assessment boards. This explains that assessment criteria change from some boards to other ones, as some true-life examples will show. Second, although the assessment process is partially regulated, it is quite ambiguous regarding the way of putting into effect the criteria. More important is the fact that assessment boards work even when they consists of four members, that is, even when three members are missing, since they would consist theoretically of seven members. These issues may generate some problems in reliability and validity. Nevertheless, these limitations can be easily rectified, therefore the national accreditation system is considered a good system since it promotes that the university positions are reached with proper and clear procedures.

Key words: UNIVERSITY PROFESSORS RECRUITMENT, NATIONAL ACCREDITATION SYSTEM, COMPETITIVE/ENTRANCE PROCEDURE.

\section{INTRODUCCIÓN}

En la historia de la Universidad española, la selección de profesores pasó por múltiples sistemas, con distintas modalidades y con distinto número de exámenes, sin embargo, siempre se mantuvo el principio de que los criterios son impuestos por los profesores que ya pertenecen a los cuerpos docentes, y que dichos criterios nunca 
fueron definidos de una forma operativa, lo cual siempre favoreció la subjetividad de los evaluadores y las críticas de los no seleccionados. Esto es una práctica tan antigua que se remonta a las primeras selecciones de profesores universitarios que se hicieron hace ya casi diez siglos en la Universidad de Bolonia (Raina y Mahoney, 2005).

En España, podría decirse que los gestores políticos de cualquier época y de cualquier ideologia, nunca fueron conscientes de lo importante que es la selección de los profesores universitarios, pues sin duda alguna, debería ser el caballo de batalla de la Universidad. Una universidad puede tener excelentes instalaciones, los mejores medios, los mejores alumnos, pero si no tiene un cuerpo de profesores con nivel de excelencia es casi lo mismo que no tener nada. La calidad del profesorado universitario es el pilar más importante para tener una buena universidad, todo lo demás puede ser importante, pero es complementario.

En la actualidad, el sistema de selección de profesorado está regulado en la Ley Orgánica de Universidades (LOU) y en el del Real Decreto 774/2002. Uno de los objetivos que se perseguian con esta Ley orgánica y con el Real decreto era mejorar el sistema de selección del profesorado funcionario, dado que la anterior Ley de Reforma Universitaria, después de dos décadas de funcionamiento, habia hecho posible un sistema tan endogámico en la selección de profesores, que si este fuese el objetivo, se convertiria en un magnifico sistema a seguir. Pero, lamentablemente, no es un buen sistema para la selección del profesorado, y así queda de manifiesto con las expresiones "el candidato de casa" y "el candidato de fuera" de uso tan frecuente que llegaron a ser normales en el lenguaje del profesorado universitario e incluso entre los miembros de las comisiones de evaluación. Se trataba de una Ley que conseguía demostrar que "el candidato de casa" siempre era "el mejor", lo cual no era difícil si tenemos en cuenta que "la casa" nombraba el $40 \%$ de los miembros de las comisiones, y además, con la excepción de "los candidatos de fuera" a nadie le interesaba que no ocurriese otra cosa que el resultado fuese conseguir la plaza "el candidato de casa", y ello implicaba desde el equipo rectoral, que en caso de encontrase con el raro hecho de que un candidato de fuera consigue una plaza, tiene el problema de que hacer con el candidato de casa, hasta el 
último profesor no numerario, que también estaba muy interesado en que la plaza siempre fuera para el candidato de casa, pues de lo contrario, podría haber un ajuste de plantilla, y con el consiguiente "efecto domino" quedarse éste sin la renovación de contrato. Así las cosas, llegó a verse como hostil el hecho de firmar una plaza en una universidad distinta de la cual el candidato trabaja. El resultado después de dos décadas de funcionamiento de este magnífico ejemplo de selección endogámica es que en algunas áreas de conocimiento y/o universidades consiguieron que en el $98 \%$ de los casos el candidato de casa conseguia la plaza. De hecho, no son raros los casos de profesores que nacieron y vivieron siempre en una misma ciudad, así, estudiaron la primaria, la secundaria y la carrera universitaria en la misma ciudad, y un día se doctoraron en esa misma universidad y luego consiguieron una plaza de profesor en la universidad de su ciudad, y para no romper la tradición repiten a sus alumnos lo que le enseñaron sus profesores. Después de tantos años en la misma ciudad y en la misma universidad se forja lo que se puede denominar como "un profesor con ralces". Es decir, se consigue justo lo contrario a lo que está explícito e implícito en el concepto de "universidad".

Por el contrario a la LRU, la LOU pretende, al menos disminuir un sistema tan endogámico en la selección del profesorado universitario, e incluye también a los profesores no numerarios. Como es bien sabido, en el sistema actual, la universidad que quiere crear o promocionar una plaza de profesor, sea para los cuerpos de funcionarios o de contratados, no tiene ninguna participación en el nombramiento de las comisiones de habilitación y de acreditación, aunque posteriormente tiene el cien por cien del control en el nombramiento de la contratación y en los concursos de acceso. Sin duda alguna, el sistema actual de selección del profesorado es, al menos en la primera fase (habilitación para funcionarios y acreditación para contratados), no endogámico, pues los miembros de las comisiones no son nombrados por quienes ofertan las plazas. En el caso de la habilitación las comisiones de evaluación están compuestas por siete miembros elegidos a sorteo de entre los profesores que cumplen un mínimo de un tramo de investigación para el cuerpo docente de Profesor Titular de Universidad y de dos tramos de investigación para 
el de Catedrático de Universidad. Por tanto, con respecto al sistema de la LRU, se mejoró: a) el cien por cien de los miembros se eligen por sorteo (frente al sesenta por cien en la LRU); b) siete miembros en la comisión (frente a cinco en la LRU); c) una mayor cualificación de los miembros de las comisiones de evaluación (uno o dos tramos de investigación frente a ninguno en la LRU); d) el lugar de la celebración de las pruebas también se determina por sorteo, la universidad en la que trabaja el presidente (frente a la universidad que convoca la plaza en la LRU).

El Sistema de Habilitación Nacional para el acceso a cuerpos de funcionarios docentes universitarios supuso una considerable mejora en la selección del profesorado, sin embargo, también presenta algunas deficiencias, y estas tienen que ver fundamentalmente con aquellos aspectos en los que se inspiró en la LRU, y en concreto se refiere a los criterios y al proceso de evaluación que utilizan las comisiones de evaluación. Por ello, en el presente artículo se describe la situación actual de selección del profesorado funcionario en España, es decir, el Sistema de Habilitación Nacional de profesores funcionarios y se analizan los criterios y del proceso de evaluación, y se revisa su puesta en práctica con algunos supuestos y con casos reales.

\section{LOS CRITERIOS DE EVALUACIÓN EN EL SISTEMA DE HABILITACIÓN NACIONAL}

Las directrices para establecer los criterios de evaluación están publicadas en el Real Decreto 774/2002, por el que se regula el Sistema de Habilitación Nacional para el acceso a cuerpos de funcionarios docentes universitarios y el régimen del Concurso de acceso respectivo (B.O.E. de 7 de Agosto, 2002). Y en concreto, éstas se recogen en:

Art. 8.6. - La Comisión fijará y, antes del acto de presentación de los candidatos, hará públicos los criterios para la valoración de las pruebas de habilitación. En dichos criterios habrá de constar con carácter preferente, aunque no exclusivo, el de la acreditación de los méritos e historial académico, docente e investigador que sean 
relevantes en la comunidad cientifica internacional y nacional del área de conocimiento de que se trate, y que constituyen el objeto de la primera prueba.

Art. 10.4.- Segunda prueba Titulares de Universidad, Catedráticos de Escuela Universitaria y Profesores Titulares de Escuela Universitaria consistirá en la (exposición oral de un tema del programa presentado por el candidato. Finalizada la prueba, cada miembro de la Comisión entregará al Presidente un informe razonado valorando los contenidos y metodología expuestos por cada uno de los candidatos.

Art. 10.5.- Segunda prueba Catedráticos de Universidad consistirá en la exposición de un trabajo original e inédito de investigación. Finalizada la prueba, cada miembro de la Comisión entregará al Presidente un informe razonado valorando los contenidos y metodología expuestos por cada uno de los candidatos.

En el Art. 8.6 se dice que los criterios para la valoración de las pruebas (nótese que se refiere a todas las pruebas y no sólo a la primera) son establecidos por la Comisión de evaluación, sin embargo, resulta muy ambiguo decir que "habrá de constar con carácter preferente, aunque no exclusivo, el de la acreditación de los méritos $e$ historial académico, docente e investigador que sean relevantes en la comunidad científica nacional e internacional del área de conocimiento de que se trate, y que constituyen el objeto de la primera prueba".

El historial académico, docente e investigador es el objeto de evaluación de la primera prueba, y esta debe tener carácter preferente, según esto, sólo cabe pensar que se refiere a las demás pruebas y/o a la exposición oral y debate de la primera prueba. Suponiendo que sea así, es excesivamente ambiguo decir "carácter preferente", pues implicaría que las demás pruebas y/o la exposición oral y debate de la primera podrían tener un valor comprendido entre el $1 \%$ y el $49 \%$ de la evaluación. Si el carácter preferente se refiere sobre las demás pruebas (dado que la exposición y debate de la primera se puede poner como un subcriterio de esta prueba), se produce el mismo problema, $y$ es que estas pueden tener un valor comprendido entre el $1 \%$ y el $49 \%$, lo cual es un margen demasiado amplio para que sea acotado por la Comisión de evaluación. Pos- 
teriormente, el Consejo de Coordinación Universitaria elaboró un modelo de Acta de Criterios de Valoración y en el que se establecen los criterios en que se fundamentará el juicio sobre los méritos de los candidatos admitidos, siendo estos:

a) Méritos relevantes en la comunidad científica nacional e internacional.

b) Historial académico relevante en la comunidad científica nacional e internacional.

c) Historial docente relevante en la comunidad científica nacional $e$ internacional.

d) Historial investigador relevante en la comunidad científica nacional e internacional.

e) Acreditación de los méritos e historial académico, docente e investigador (carácter preferente no exclusivo).

Es importante destacar que en el último criterio, tal como está definido en el Documento 03 Acta de Criterios de Valoración de la Prueba de Habilitación, genera mucha confusión, pues al decir "carácter preferente no exclusivo" en uno de los criterios, lógicamente las comisiones entienden que se refiere a que ese criterio debe ser referente sobre los cuatro anteriores, lo cual es un error, pues el Real Decreto 774/2002 lo que dice es que el carácter preferente lo tienen: los méritos e historial académico, docente e investigador, es decir, los cuatro criterios anteriores, y que constituyen el objeto de la primera prueba. De hecho, el criterio cinco incluye a los cuatro anteriores, no es un criterio más, tal como parece que se ha interpretado por el Consejo de Coordinación Universitaria.

Es evidente que los criterios anteriores son tan ambiguos que dependen de la interpretación que haga cada Comisión evaluadora. Así, por ejemplo, no se menciona si el acto de "Exposición y detensa oral" debe ser evaluado y en que medida sobre la evaluación total. $Y$ una buena muestra es que mientras que en algunos casos como por ejemplo, en la Habilitación Nacional al Cuerpo Docente de Catedráticos de Universidad del área de conocimiento de Personalidad, Evaluación y Tratamientos Psicológicos (Código 1/680/0902), se estableció como un subcriterio para cada uno de los cinco criterios antes mencionados, en otros, como por ejemplo, en la Habilitación Nacional al Cuerpo Docente de Catedráticos de Universidad del área 
de conocimiento de Psicología Básica (Código 1/730/01013), no se hace mención alguna a la "Exposición y defensa oral".

Otra muestra de la ambigüedad de los criterios se puede comprobar en la interpretación que hacen de un mismo criterio dos comisiones de evaluación de Psicología, véase qué méritos consideran dos comisiones con respecto al criterio $\mathrm{E}$ (Acreditación de los méritos e historial docente e investigador):

Comisión 1: Habilitación Nacional al Cuerpo Docente de Catedráticos de Universidad del área de conocimiento de Personalidad, Evaluación y Tratamientos Psicológicos (Código 1/680/0902), en el criterio $\mathrm{E}$ (Acreditación de los méritos e historial docente e investigador) incluyen como subcriterios:

- Complementos docentes.

- Complementos de investigación

- Otras acreditaciones

- Exposición y defensa oral de la acreditación

Comisión 2: Habilitación Nacional al Cuerpo Docente de Catedráticos de Universidad del área de conocimiento de Psicologia Básica (Código 1/730/01013), en el criterio E (Acreditación de los méritos e historial docente e investigador) incluyen como subcriterios:

- Todos los méritos académicos alegados se acreditan mediante certificación reglamentaria correspondiente.

- Las aportaciones cientificas preferentemente valoradas serán artículos en revistas que cuenten con comité editorial formado por científicos de reconocido prestigio, y con un riguroso proceso de evaluación de manuscritos. Se tendrán en cuenta los índices de impacto de las revistas o las citas del manuscrito en dichas revistas, como criterio de ponderación de las publicaciones.

- Se tendrán en cuentan los libros y capítulos de libro publicados por editores de reconocido prestigio.

- Asimismo se valorarán estudios, informes o dictámenes que estén planteados con rigor científico, publicados por la Institución que lo haya solicitado.

Es un hecho evidente, que las dos comisiones interpretan el criterio sobre "Acreditación de los méritos e historial docente e investigador" de forma muy distinta e incluyen subcriterios muy distintos, y muestra de ello es que no hay ni la más mínima coincidencia 
en cuanto a los subcriterios que establece cada comisión para el mismo criterio. Pero se dan casos aun más sorprendentes, pues en un mismo criterio, se pueden proponer incluso subcriterios totalmente opuestos. Por ejemplo, mientras que en la Habilitación Nacional al Cuerpo Docente de Catedráticos de Universidad del área de conocimiento de Psicología Básica (Código 1/730/0103), en el criterio E se establece un subcriterio: Se tendrán en cuentan los libros y capítulos de libro publicados por editores de reconocido prestigio; en la Habilitación Nacional al Cuerpo Docente de Catedráticos de Universidad del área de conocimiento de Psicología Básica (Código 1/730/0102), en el criterio $E$ se establece un subcriterio: No se tomarán en consideración los libros de texto, o las traducciones, los apuntes, o los meros artículos de opinión. Es importante resaltar que estas dos comisiones pertenecen a una misma área de conocimiento.

En cuatro de los cinco criterios se dice "relevantes en la comunidad científica nacional e intemacional', esto es muy ambiguo pues no se dice que se entiende por comunidad científica internacional, por ejemplo, ¿publicar un artículo en una revista francesa es un mérito internacional?, y si se considera como intemacional la producción científica de distintos países, la producción científica nacional no forma parte de la internacional. El concepto de producción científica internacional no esta suficientemente delimitado, véase Buela-Casal (2001), y de igual manera, no está claro qué es una publicación internacional si se limita a la opinión personal del evaluador, y sin embargo, si es posible crear un índice para su medida, tal como por ejemplo el propuesto por Buela-Casal, Perakakis, Taylor y Checa (2005). Además, en el ámbito de la Psicología, incluso hay costumbres claramente distintas entre Europa y Estados Unidos con relación a la gestión y dirección de las revistas, pues mientras que las revistas europeas se tiende a mantener la dirección y el comité editorial durante muchos años, en las revistas estadounidenses se cambia de manera periódica tanto el comité editorial como la dirección (Pelechano, 2000), y estos dos modelos sin duda tienen su influencia en la aceptación de artículos. En resumen, que ni se dice qué se entiende por internacional, ni se dice qué debe valer más, todo queda según el propio criterio de cada Comisión evaluadora, que 
dicho sea de paso, se le presentarán las mismas dudas antes mencionadas, dado que al igual que se puede defender que una publicación tenga más valor por su difusión internacional, también se pueden dar importantes argumentos para valorar la adaptación de la investigación psicológica a la cultura española. Una interesante reflexión sobre esto puede verse en Pérez (2002), o también en Pelechano (2005b) donde se propone que incluso los contenidos del título de Licenciado en Psicología deben estar diversificados en función del nicho histórico-cultural en el que se imparten las enseñanzas, lo cual incluso debe tenerse en cuenta en los programas de doctorado (Pelechano, 2005a).

En el Art. 10, apartado 3, se dice: "Finalizada la prueba, cada miembro de la Comisión entregará al Presidente un informe razonado, ajustado, en todo caso, a los criterios previamente establecidos por la Comisión, valorando los méritos e historial académico, docente e investigador... A la vista de los informes, la Comisión procederá a una votación, no siendo posible la abstención, para determinar el paso de los candidatos a las pruebas sucesivas. No pasarán a la siguiente prueba los candidatos que no obtengan, al menos, cuatro votos favorables". El problema principal aquí es que no se sabe en función de que nivel de los criterios se debe votar favorable o desfavorable. Si tenemos en cuenta que en este ejercicio no hay un límite de candidatos con cuatro o más votos, en principio es razonable decir que el nivel no está en el promedio de los candidatos presentados, ni tampoco en función de los que mejor curriculum tienen. Los miembros de la Comisión tienen unos criterios que han establecido, pero nada se dice sobre qué nivel o umbral debe alcanzar un candidato para superar este ejercicio, y así, una vez más queda al criterio personal de cada miembro de la Comisión. Este problema no existía en la anterior Ley de Reforma Universitaria, pues estaba regulado que el primer ejercicio debe tener un valor como mínimo doble y como máximo triple que el segundo ejercicio, pues en este caso la puntuación del candidato con mejor curriculum si determinaba en gran medida la puntuación que obtienen los otros, pero en la actual Reglamento, al decir tan sólo que es preferente no se produce este efecto de comparación necesaria de los curricula de los candidatos. Por otra parte, cada miembro de la Comisión no 
tiene una información objetiva sobre cual es el nivel necesario para superar este ejercicio, pues la información que dispone se puede basar en: el nivel que él tenía cuando consiguió la plaza; o con el que tienen los profesores de su entorno; o con el nivel que tenían otros candidatos que evaluó en otras oposiciones. Sea cual sea de los anteriores, es difícil defender que es una buena forma de obtener el nivel que deben tener los candidatos. Decir, por ejemplo, que 50 artículos, 5 tesis doctorales y 5 libros, etc. es el nivel necesario para superar la primera prueba de Habilitación de Catedráticos de Universidad, es tan válido como decir que no lo es, pues en ambos casos se trata de una opinión personal. Para un buen funcionamiento del actual Sistema de Habilitación Nacional en el cual los candidatos compiten a un nivel nacional, es necesario disponer de información sobre la productividad científica media de los investigadores españoles, algunos ejemplos son: el estudio de Agudelo, Bretón-López, Ortiz-Recio et. al. (2003) sobre la productividad científica de la Psicología española a través de las tesis doctorales; el análisis de la productividad de las universidades en función de los tramos de investigación y su relación con otros criterios de productividad (BuelaCasal, 2005); o el trabajo de Gordillo, Gonzáles-Marqués y Muñiz (2004) sobre la evaluación de proyectos de investigación por la ANEP.

En el Art. 10.4. del Real Decreto 774/2002 se dice que la "segunda prueba para Profesores Titulares de Universidad, Catedráticos de Escuela Universitaria y Profesores Titulares de Escuela Universitaria consistirá en la exposición oral de un tema del programa presentado por el candidato. Finalizada la prueba, cada miembro de la Comisión entregará al Presidente un informe razonado valorando los contenidos y metodología expuestos por cada uno de los candidatos". En este criterio, lo único que se establece es que hay que evaluar los contenidos y la metodología expuestos, pero no se dice ni cómo ni en función de qué, y según esto, una vez más queda la decisión al total arbitrio de la Comisión evaluadora, que, además, no comunica previamente a los candidatos cual es la metodología adecuada para ser bien evaluado, no se sabe a priori, si es mejor usar poco o mucho apoyo de medios técnicos, si es mejor usar medios clásicos o modernos, si es mejor presentarlo sentado o de 
pié, si usar un estilo muy técnico y académico o un estilo más ameno y accesible para estudiantes de primeros cursos, etc. Además, el sistema de presentación del tema no permite más que distintas variantes de una clase magistral, pero sin saber si debe representarse como una clase para alumnos o una exposición para el tribunal, lo cual, sin duda, determina los contenidos que se presenten, el estilo y el nivel académico. En realidad, no hay criterios más allá de la opinión y/o el gusto personal de cada miembro de la Comisión evaluadora.

En el Art. 10.5. del Real Decreto 774/2002 se dice que la "Segunda prueba para Catedráticos de Universidad consistirá en la exposición de un trabajo original e inédito de investigación. Finalizada la prueba, cada miembro de la Comisión entregará al Presidente un informe razonado valorando los contenidos y metodología expuestos por cada uno de los candidatos". Al igual que en el caso anterior, los candidatos no tienen ninguna información adicional sobre qué forma de exposición es la más adecuada o la mejor valorada. Pero, además, en este caso, al tratarse de un trabajo original de investigación, el nivel de ambigüedad es aun mayor que en el criterio anterior, pues en primer lugar puede darse una importante discrepancia en cuanto a la originalidad de un trabajo, y en segundo lugar, los candidatos no saben que tipo de metodología es la mejor valorada por parte del tribunal, según la reciente Clasificación de los métodos de investigación propuesta por Montero y León (2005) podría hablarse de hasta nueve tipos distintos de investigación según el método empleado. Suponiendo que los candidatos tengan más de una opción entre sus investigaciones ya realizadas, no sabrian cuál será mejor valorada, por ej. ¿es mejor un estudio experimental con alta validez interna que uno instrumental con alta validez externa?. Y por último, en cuanto a la metodología expuesta, tampoco se hace explicita ninguna pauta sobre el tipo de presentación mejor valorada, y sin embargo, si existen protocolos detallados que permiten una exhaustiva evaluación de los múltiples aspectos que se pueden valorar de un trabajo de investigación y de su presentación, véase por ejemplo, Buela-Casal, López y Fernández-Hermida (2002) y Ramos-Álvarez y Catena (2004). 


\section{EL PROCESO DE EVALUACIÓN DEL SISTEMA DE HABILITACIÓN NACIONAL}

El proceso de evaluación está recogido en el Real Decreto 774/ 2002, por el que se regula el Sistema de Habilitación Nacional para el acceso a cuerpos de funcionarios docentes universitarios y el régimen del concurso de accesos respectivos (B.O.E. de 7 de Agosto, 2002), pues se establece el proceso a seguir para cada una de las pruebas y para el funcionamiento de la Comisión evaluadora para ejercer su función. Sin embargo, si se analizan los articulos relativos al proceso, nos encontramos que éstos son muy pocos y a la vez muy limitados pues no son suficientes para guiar la actividad de la Comisión evaluadora. En concreto, se pueden considerar como proceso los siguientes:

Artículo 8: Constitución de las Comisiones y convocatoria, tiene ocho apartados, y todos excepto el seis, ya mencionado anteriormente y que se refiere a los criterios, regulan el funcionamiento de la Comisión, pero no se relacionan con la forma de evaluar. No obstante, es especialmente importante resaltar el punto 4 de este artículo: "Para que la Comisión pueda actuar válidamente será necesaria la participación de, al menos, cuatro de sus miembros". Es decir, que una Comisión puede actuar con siete, con seis, con cinco, y con cuatro miembros, pero en todos los casos los candidatos siempre necesitan un mínimo de cuatro votos tanto para superar un ejercicio como para ser habilitado, y esto es especialmente importante pues el hecho de ser acreditado depende, entre otras variables, del número de miembros que hay en la Comisión, lo cual lógicamente no debería tener ningún efecto sobre el resultado de la evaluación de los candidatos. Sin embargo, esto tiene un gran efecto, tanto desde la perspectiva del porcentaje de decisión de cada miembro del tribunal como del porcentaje de votos positivos que necesita un candidato para superar un ejercicio o para ser habilitado. En la figura 1 se muestra como el porcentaje de decisión de cada miembro de la Comisión varia en función del número de miembros que componen la Comisión, asi, este porcentaje oscila desde un $14 \%$ cuando hay siete miembros, hasta un $25 \%$ cuando hay 4 miembros, y que además se convierte en "voto clave" pues cuando sólo hay cuatro miembros 
en la Comisión, el voto de cada miembro es necesario para que pase o se habilite un candidato. Desde la perspectiva de los candidatos se produce un efecto similar, por ejemplo, cuando hay siete miembros en el tribunal, un candidato supera una prueba o es habilitado si consigue el $57 \%$ de los votos favorables (4 de 7 ), mientras que este porcentaje se incrementa, tal como se puede ver en la figura 2 , hasta un $100 \%$ cuando sólo hay cuatro miembros en el tribunal. Esto es especialmente importante si tenemos en cuenta que un candidato con el $57 \%$ de los votos favorables supera una prueba cuando hay siete miembros en la Comisión y no la supera con el $75 \%$ de los votos favorables cuando hay cuatro miernbros en la Comisión.

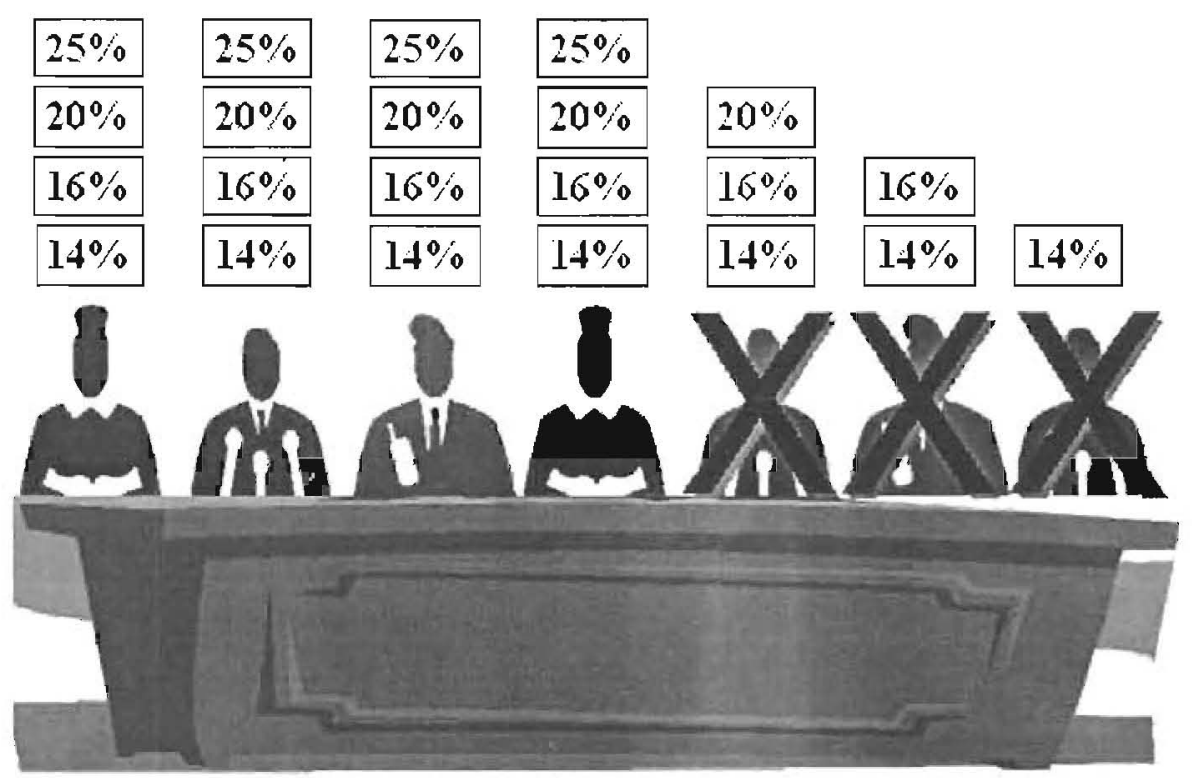

Figura 1.- Comisión con siete, seis, cinco y cuatro miembros 

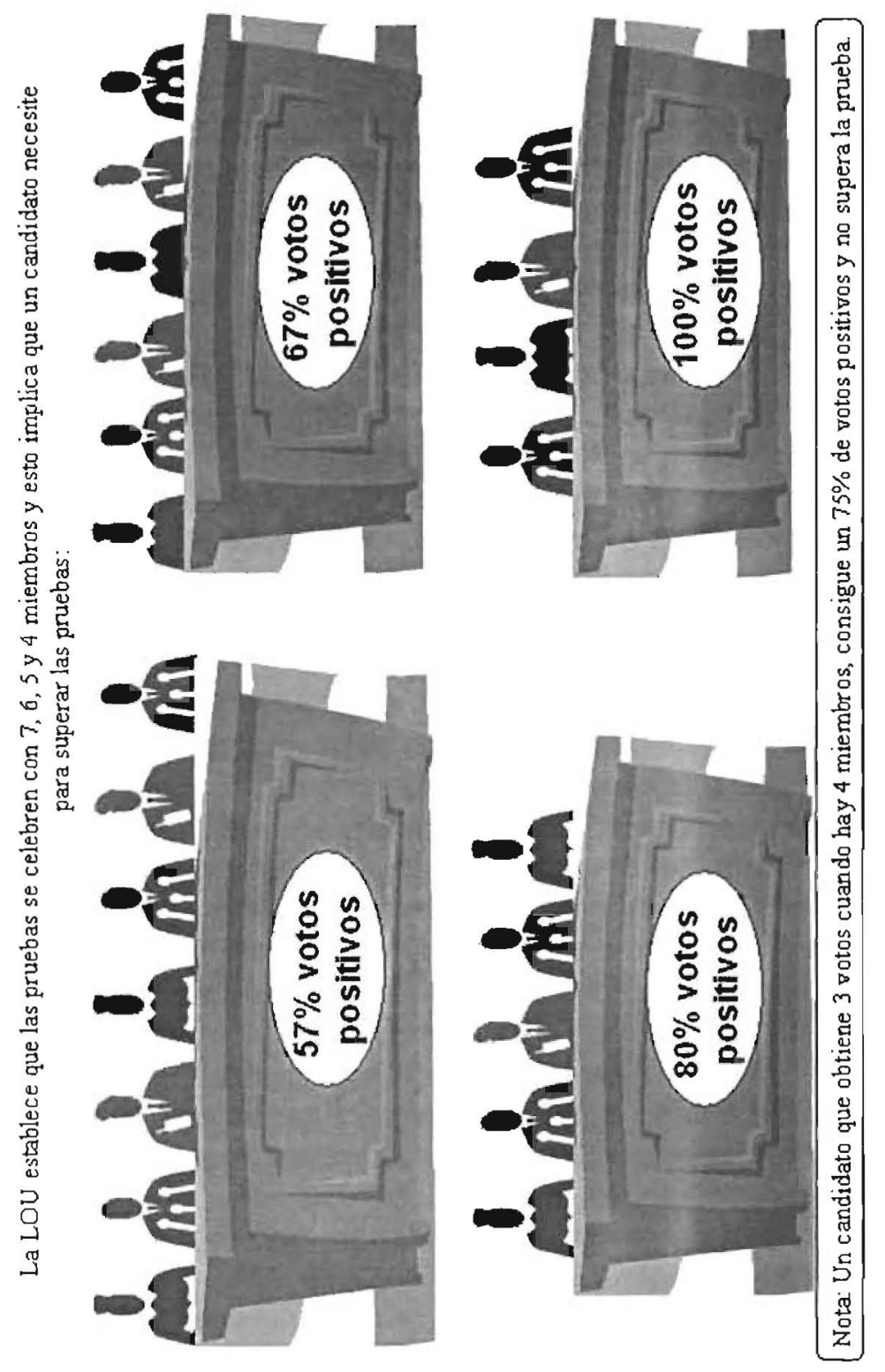

ป5 
Articulo 9: Acto de presentación de los candidatos, se refiere fundamentalmente a las condiciones de presentación de los candidatos, orden de presentación, plazos, documentación, etc., pero no contiene ninguna información sobre el proceso técnico de la evaluación.

Artículo 10: Celebración de las pruebas, se recogen varios apartados sobre el funcionamiento, como por ejemplo el tiempo máximo establecido para la presentación de los ejercicios por parte de los candidatos (90 minutos para la primera prueba) y para el debate entre la Comisión y el candidato (120 minutos). Con respecto al tiempo máximo del debate hay que resaltar que es muy limitado, dado que al haber siete miembros en la Comisión, si se reparte el tiempo de forma equitativa, cada miembro de la Comisión tiene 6 minutos para preguntar y el candidato otros 6 para responder. Esto supone que como mucho cada miembro de la Comisión puede hacer dos o tres preguntas, teniendo así el candidato de 2 a 3 minutos para contestar cada pregunta. Por ello, es más que sorprendente ver que en algunos casos, como por ejemplo, en la Habilitación Nacional al Cuerpo Docente de Catedráticos de Universidad del área de conocimiento de Personalidad, Evaluación y Tratamientos Psicológicos (Código 1/680/0902), se llegó a establecer como un subcriterio "la exposición oral y la defensa" en cada uno de los cinco criterios propuestos por el Consejo de Coordinación Universitaria, ya mencionados anteriormente. En este ejemplo, que se trata de un caso real, es al menos difícil de entender como los miembros del tribunal pueden asignar un valor a una defensa oral como un subcriterio, cuando el tiempo máximo de la defensa por parte del candidato no puede ser más de 1 minuto y 12 segundos (que es el resultado de dividir los 6 minutos entre los cinco subcriterios), y no debe olvidarse que el informe razonado debe hacerse en función de los criterios y subcriterios propuestos por la propia Comisión, por tanto, no se trata de una cuestión baladí. La conclusión es que con un tiempo tan limitado para el debate es difícil defender que el debate con el candidato pueda ser relevante en la decisión final. En el mismo artículo se establece que "A la vista de los informes, la Comisión procederá a una votación, no siendo posible la abstención para determinar el paso de los candidatos a las pruebas sucesivas. No 
pasarán a la siguiente prueba los candidatos que no obtengan, al menos, cuatro votos favorables". Una vez más no se establece el procedimiento para otorgar los votos favorables o desfavorables, todo depende de la opinión personal de cada miembro de la Comisión en función de los criterios previamente establecidos. Es decir, no se sabe si se vota en función del grupo normativo, en función de un criterio externo o una opinión personal. $Y$ esto se relaciona con lo siguiente.

Art. 10.5.- La Comisión procederá a la votación, sin que sea posible la abstención, teniendo en cuenta que no podrán proponer como habilitados a más candidatos que el número de habilitaciones que hayan sido objeto de la convocatoria. Esto implica que no podrá obtener más de cuatro votos favorables un número de candidatos mayor que el número de habilitaciones. En este caso, es evidente que el voto de cada miembro de la Comisión está determinado, entre otros criterios, por el número de candidatos, pero también puede votar en función de una opinión personal del nivel que debe tener un candidato para ser habilitado. La ambigüedad del proceso es tal que permite, y en algunas ocasiones ocurre, que un miembro de la Comisión otorga a un mismo candidato un voto desfavorable en el primer ejercicio y un voto favorable en el segundo, esto que obviamente es incongruente, lo permite el Sistema de Habilitación. Pero esto no es el único problema, pues el sistema obliga a los miembros de la Comisión a pactar las votaciones. Dado que no pueden proponerse más candidatos con cuatro votos que plazas ofertadas, esto implica que cada miembro de la comisión no puede votar de forma independiente por los candidatos que considere adecuados. Por ejemplo, en una oposición con dos plazas, con siete miembros en el tribunal y siete candidatos, si cada miembro de la Comisión vota de forma independiente se puede dar el caso de siete candidatos con cuatro votos, tal como se muestra en la tabla 1.

Por tanto, el sistema obliga a los miembros de la Comisión a pactar las votaciones, pero no se establece cómo ni en función de qué unos miembros de la Comisión deben cambiar sus votos en detrimento de otros, hasta que se consiga que no se obtengan más candidatos con cuatro o más votos que las plazas en concurso. Otra opción es que la Comisión acuerde que cada miembro pueda votar 
por un número de candidatos inferior al número de plazas, de tal forma que no se puedan obtener nunca más candidatos con cuatro votos que plazas hay en concurso. Sin embargo, esta no es una buena solución, pues se limita el número de votos que puede otorgar un miembro de la Comisión, y puede darse caso de que queden plazas vacantes cuando hay candidatos que recibirian al menos cuatro votos, si no hubiese tal restricción en el proceso de votación.

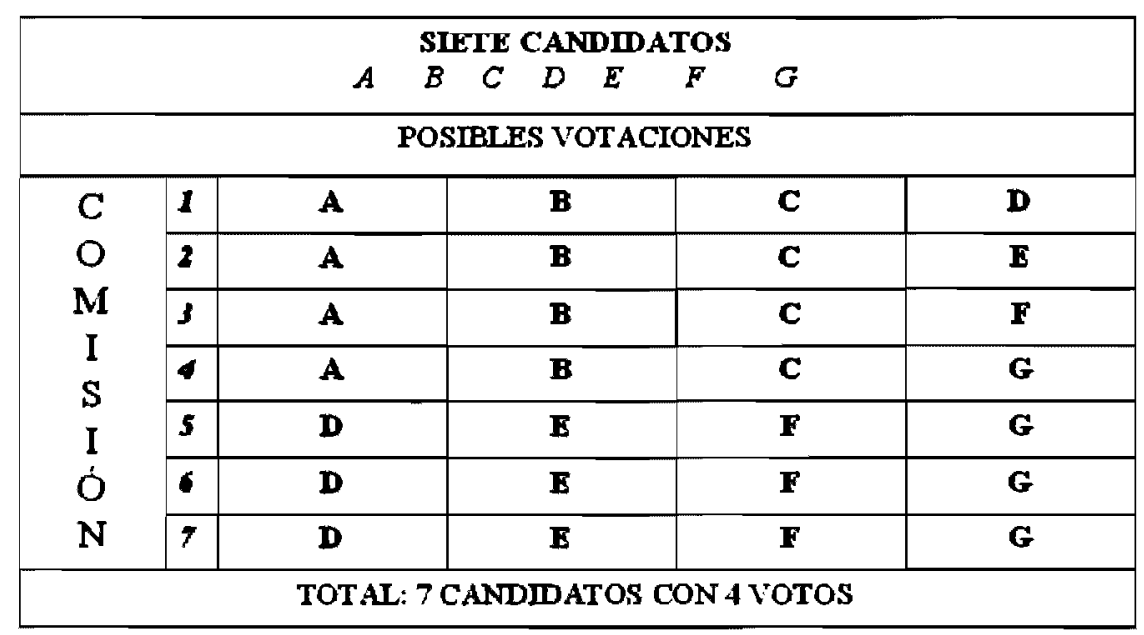

Tabla 1. Sistema de Votación en la Habilitación Nacional

\section{VALIDEZ Y FIABILIDAD DE LA EVALUACIÓN EN EL SISTEMA DE HABILITACIÓN}

Los distintos problemas relacionados con los criterios y con el proceso de evaluación comentados anteriormente afectan a la validez y a la fiabilidad de la evaluación. Si partimos del hecho de que no se determina a priori los criterios y el nivel de los criterios necesarios para ser habilitado, y si además no existe un proceso de evaluación reglado que guíe la actuación de la Comisión de evaluación, es lógico que se presenten problemas de validez y fiabilidad. A continuación se comentan algunos aspectos relacionados con esto.

- Variabilidad en los criterios establecidos por las comisiones de evaluación 
En el apartado de criterios ya se puso de manifiesto la arbitrariedad con la que se puede interpretar los criterios propuesto por el Consejo de Coordinación Universitaria, y se han puesto algunos ejemplos claros en los que se establecen subcriterios distintos según sea la Comisión de evaluación. Por tanto, si no están regulados los subcriterios, quedando estos al arbitrio de cada Comisión, es evidente que esto afecta a la validez y fiabilidad de la evaluación.

- La evaluación de la calidad de los curricula de los candidatos

Con relación a los curricula de los candidatos es cuestionable que la Comisión de evaluación pueda evaluar la calidad de los curricula, y ello por dos razones: a) la calidad ya fue evaluada previamente por otros evaluadores más especializados; b) dado el tiempo disponible no es posible leer el material aportado por los candidatos. Esto queda de manifiesto en el supuesto recogido en la tabla 2, donde se sintetiza un curriculum imaginario de un candidato a la habilitación del cuerpo de Catedráticos de Universidad.

\begin{tabular}{|l|l|}
\hline 5 Libros X 300 págs. & 1.500 págs. \\
\hline 50 Artículos X 10 págs. & 500 págs. \\
\hline 30 Capihulos de libro X 20 págs. & 600 págs. \\
\hline 50 Congresos y otros documentos & 200 págs. \\
\hline 5 Proyectos de investigación & \\
\hline 5 Tesis & \\
\hline TOTAL & 2.800 páginas \\
\hline
\end{tabular}

Tabla 2. Curriculum de un candidato medio

En el caso supuesto descrito en la tabla 2 se muestra como para un sólo candidato la Comisión de evaluación se ericuentra con que tendría que leer aproximadamente unas 2.800 páginas. Esto implica 
que si se presentan veinte candidatos la cantidad sería 56.000 páginas, lo cual es evidente que no es posible que sea leído por los miembros de las Comisiones de evaluación. Así, es un hecho indiscutible que ningún miembro de las comisiones de evaluación lee las publicaciones de los candidatos, y de ahí, que en algunas situaciones se producen situaciones bochornosas para algun miembro, por ejemplo, un caso muy conocido en una oposición de Psicología es el de un candidato que fue criticado por un miembro del tribunal por no aplicar el procedimiento de la traducción y retrotraducción en la adaptación de un test, a lo cual el candidato contestó que si el miembro del tribunal se hubiese molestado en abrir el test (que tenía encima de la mesa y aun con el precinto) se hubiese percatado de que no tenía elementos lingüísticos, puesto que los ítems eran figuras.

Por otra parte, si se analiza como se evalúa la producción científica de un investigador para que ésta pueda convertirse en publicaciones, congresos o tesis, se puede comprobar que fue evaluado previamente por una gran cantidad de evaluadores especializados. Si tenemos en cuenta que para que un libro se publique es evaluado al menos por un especialista (el asesor o director de la colección) y por un responsable de la editorial que estima la posible demanda de ese libro; un artículo es evaluado al menos por dos revisores especializados más el director de la revista que también evalúa los informes de los anteriores; un capítulo de un libro es evaluado al menos por el director del libro; una presentación en un congreso es seleccionada al menos por un miembro del comité científico; un proyecto de investigación es evaluado al menos por dos investigadores especializados en el tema; una tesis doctoral es evaluada por cinco doctores especializados. Por tanto, es evidente que los méritos de un candidato fueron evaluados por un gran número de especialistas que se leyeron la totalidad de los trabajos. Siguiendo con el ejemplo supuesto mencionado antes, en la tabla 3 se muestra el número de especialista que previamente evaluaron al candidato, y como se puede ver, unos 275 evaluadores especializados habian leído y evaluado previamente la calidad de la producción científica de ese candidato. Si suponemos que se presentan veinte candidatos similares, el resultado, como se puede ver en la tabla 4, es que esos candidatos habían sido evaluados previamente por 5.500 evaluadores especializados que si leyeron los 
trabajos. Según esto, es difícil defender que los miembros de una Comisión de evaluación pueden dar una opinión más válida y fiable sobre la calidad de los méritos evaluados que los evaluadores previos, que además eran especializados.

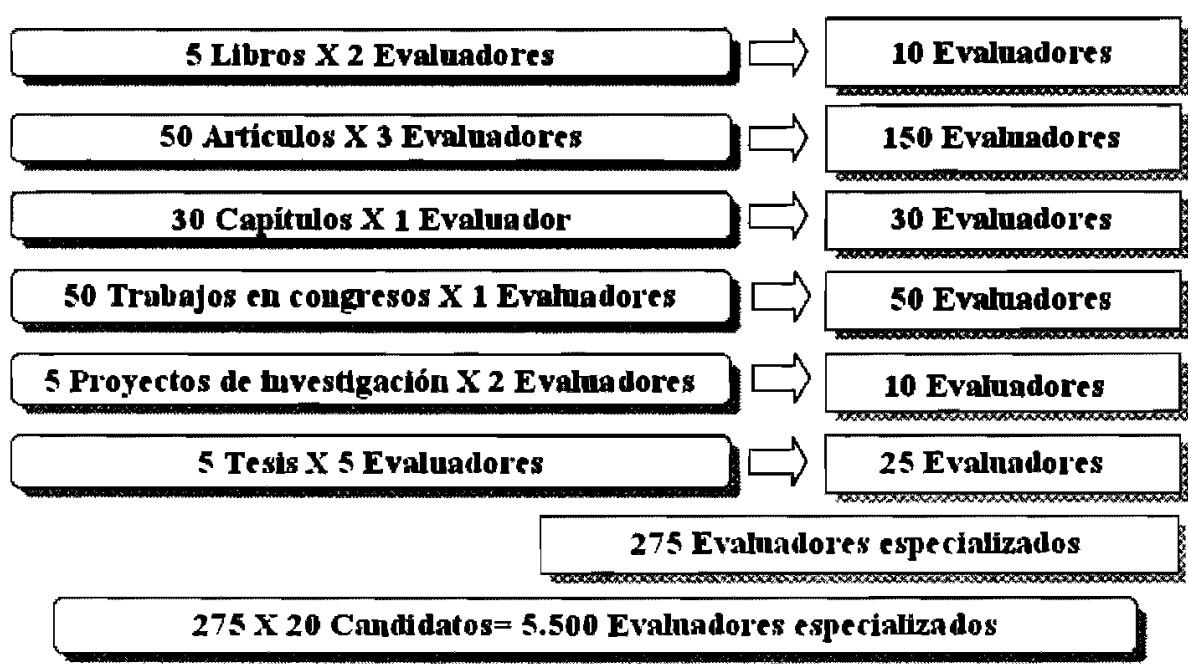

Tabla 3. ¿Quién evaluó previamente a los candidatos?

- La evaluación de la cantidad del curriculum de los candidatos.

Las comisiones evalúan en alguna medida la cantidad de los méritos de los candidatos, pero sin duda, el problema es que al no estar establecido un criterio de referencia externo, en la primera prueba, en la que pueden pasar desde ninguno hasta todos los candidatos, el criterio de referencia de los miembros de la Comisión de Evaluación pueden ser varios: a) los méritos que él tenía cuando entró en ese cuerpo; b) los méritos que él tiene en la actualidad; c) el "promedio subjetivo" de méritos de los miembros que conoce de ese cuerpo; d) el nivel medio de los candidatos evaluados en 
concursos/oposiciones anteriores; e) un nivel ideal que cree debe cumplir un profesor para ingresar en ese cuerpo; etc. Según esto, es fácil explicar por que unos miembros de las comisiones defienden que lo más importante es la gestión universitaria, para otros lo es la docencia, otros mantienen que la publicación de artículos en revistas del ISI, para otros la publicación de libros, etc. Aunque no existen estudios al respecto, parece que lo más habitual es que los miembros del tribunal tienden a valorar aquel curriculum que más se asemeja al propio, quizá para evitar así una disonancia cognitiva, por ejemplo es difícil que un miembro de un tribunal que no dirigió tesis doctorales, valore la dirección de tesis como un mérito importante. Esto queda claramente de manifiesto en el viejo dicho "lo importante y principal el conocer bien al tribunal, y lo secundario, conocer algo el temario". Por tanto, la evaluación de la cantidad de méritos de los candidatos se realiza en función de distintos criterios y niveles, lo cual sin duda afecta a la validez y fiabilidad de le evaluación.

- La evaluación de los conocimientos de los candidatos.

Al tratarse de un concurso-oposición es evidente que a priori se espera que los miembros de la Comisión evalúen también conocimientos y destrezas de los candidatos, pero tal como se explicó anteriormente, dado el tiempo tan limitado para el debate con los candidatos, difícilmente se puede evaluar, pues que como ya se explicó, cada miembro del tribunal no puede formular más de dos o tres preguntas a cada candidato. Incluso así, tampoco existe un procedimiento que trate de garantizar que las preguntas formuladas a los distintos candidatos tengan un nivel de dificultad similar.

Según lo anteriormente expuesto es previsible que se produzcan importantes problemas de validez y fiabilidad entre las evaluaciones realizadas por distintas comisiones sobre los mismos candidatos, y algunos ejemplos reales son los siguientes:

Ejemplo 1: Si se comparan los resultados obtenidos en el primer ejercicio de dos habilitaciones nacionales al Cuerpo Docente de Catedráticos de Universidad del área de conocimiento de Personalidad, Evaluación y Tratamientos Psicológicos, códigos: 1/680/0902 y $1 / 680 / 0903$ se puede comprobar que hay nueve candidatos comunes que se presentaron a las dos oposiciones, y de estos, una vez 
ajustados los datos en función de los miembros de las comisiones (la primera actuó con siete miembros y la segunda con seis) el resultado es que tan sólo un tercio de los candidatos reciben el mismo número de votos. Pero más importante es aun si se analiza como el número distinto de votos afectó realmente a los candidatos para pasar o no la prueba, y el resultado es que dos de los candidatos que pasaron la primera prueba en la primera habilitación no lo superaron en la segunda, y hay que resaltar que entre una y otra hay un periodo de un año y medio.

Ejemplo 2: Al comparar los resultados obtenidos en el primer ejercicio de dos habilitaciones nacionales al Cuerpo Docente de Catedráticos de Universidad del área de conocimiento de Psicología Básica, códigos: 1/730/0102 y 1/730/0103, se producen resultados similares al caso anterior, pues si se analizan los votos favorables que reciben los candidatos que se presentan a las dos habilitaciones, y una vez ponderados los datos, dado que no hay el mismo número de miembros en las dos habilitaciones, se pone de manifiesto que en el $75 \%$ de los casos no reciben los mismos votos, y el efecto real de superar o no la prueba no coincide en el $25 \%$ de los casos. Pero en estas dos habilitaciones es especialmente importante resaltar el caso del candidato A.C.O. que en la primera habilitación no supera la primera prueba con siete miembros en la Comisión, mientras que en la segunda, no sólo supera la primera prueba, sino que además consigue ser uno de los dos habilitados. Es decir, con respecto a la primera prueba de la primera habilitación consiguió el $42 \%$ de los votos favorables, en la segunda habilitación, celebrada unos meses después consiguió el $80 \%$ de los votos favorables. Pero sin duda, el hecho más llamativo es que un candidato que no supera la primera prueba con una Comisión, meses después consigue la Habilitación concursando con varios candidatos comunes en las dos habilitaciones.

Los dos ejemplos anteriores son una buena muestra de los problemas de validez y fiabilidad de la evaluación realizadas por algunas comisiones de evaluación, lo cual era previsible según lo comentado en este apartado. Por ello, es lógico pensar que el sorteo de los miembros de las comisiones supone un alto porcentaje de varianza explicada con respecto al resultado final de una Habilitación, y de ahí la gran expectación que genera el sorteo de las co- 
misiones para los candidatos, y ello hasta tal punto que es una práctica habitual "firmar la habilitación y esperar el resultado del sorteo, y en función de ello se toma la decisión de presentarse”. Lo cual es hasta cierto punto lógico dado el importante efecto que tiene el resultado del sorteo sobre el resultado de la Habilitación, pero al mismo tiempo, es un claro indicador de la falta de fiabilidad y de validez del sistema de evaluación.

Es evidente y también de sentido común que de unos casos particulares no se puede generalizar y decir que el sistema carece totalmente de validez y fiabilidad, pues sin duda, habrá muchos casos en los que la validez y fiabilidad se cumple a rajatabla a través de distintas comisiones e incluso actuando bajo distintas leyes, es por ejemplo el siguiente caso:

Ejemplo 3: Es el caso de una candidata que se presentó a una convocatoria de un programa de dotación de cátedras de su universidad, en la que la Comisión de selección debía reunir además de los requisitos de excelencia académica, el de que nunca un candidato tuviese que ser evaluado por alguien de la misma área de conocimiento que el candidato. El resultado fue que esta candidata no fue seleccionada, sin embargo, posteriormente, sí pudo concursar a una plaza de cátedra de su área de conocimiento, pues su Universidad había dotado una cátedra (estando aun vigente la LRU) en función de otro candidato que si habia sido seleccionado. Una vez celebrado el concurso oposición esta candidata no consigue superar la primera prueba y presenta una reclamación a su Universidad, la Comisión de reclamación de su Universidad (en la que no hay ningún miembro del área de conocimiento de la candidata) concluye que no procede la reclamación, validando así la resolución de la Comisión de evaluación. Posteriormente esta candidata se presenta a una Habilitación del cuerpo de Catedráticos de Universidad y el resultado es que tampoco consigue superar la primera prueba. Sin duda, este es un caso ejemplar de validez y fiabilidad de las comisiones de evaluación, pues cuatro comisiones de evaluación distintas siguiendo normativas de dos leyes universitarias (LRU y LOU), criterios y procesos distintos, llegan exactamente a la misma conclusión. 
Los ejemplos anteriores ponen de manifiesto que hay argumentos tanto para aquellos que defienden unos criterios y proceso de evaluación estrictos y reglados como para aquellos que defienden la autonomía del "criterio sabio" de la Comisión, que independientemente de la ley, proceso y criterios saben seleccionar a los candidatos más idóneos en cada caso. Pero quizás el problema principal para aplicar el "criterio sabio" es que no se pueden seleccionar "sabios" con el criterio de un tramo de investigación para las habilitaciones a Profesor Titular de Universidad y con dos tramos de investigación a Catedráticos de Universidad.

\section{LOS CONCURSOS DE ACCESO A CUERPOS FUNCIONARIOS DOCENTES UNIVERSITARIOS}

Los concursos de acceso a cuerpos de funcionarios también están regulados por el Real Decreto 774/2002, no obstante, con excepción de los plazos que se deben cumplir y los trámites para su resolución, los criterios y el proceso de evaluación, e incluso la constitución y la composición de la Comisión están determinados por los estatutos de cada universidad. La única directriz que se establece a las universidades con relación al concurso se recoge en el apartado 3 del Art. 14: "La convocatoria, realizada por el Rector, determinará las plazas objetos de concurso, señalando la categoría del cuerpo, el área de conocimiento a que pertenecen $y$, en su caso, las actividades docentes e investigadoras referidas a una materia de las que se cursen para la obtención de títulos de carácter oficial de primero y segundo ciclo que deberá realizar quien obtenga la plaza. La existencia de dichas especificaciones, en ningún caso, supondrá, para quien obtenga la plaza, un derecho de vinculación exclusiva a esa actividad docente e investigadora, ni limitará la competencia de la Universidad para asignarle distintas obligaciones docentes $\theta$ investigadoras". Esto es casi lo mismo que decir a las universidades, "tienen ustedes patente de corso para el concurso de acceso, pero no tienen ningún compromiso después". Es importante considerar que sólo se obliga a que las actividades docentes se refieran a una materia, según lo cual, se podría poner una materia que impartiese 
"el candidato de casa" y consecuentemente el mérito lo tendría asegurado aquel candidato que trabaja en la universidad que convoca la plaza, lo cual no ocurriría si se limitase a materias del área de conocimiento. Lo mismo ocurre con la actividad investigadora. Por ello, es posible que las universidades pongan perfiles docentes y de investigación tan específicos como quieran, y un buen ejemplo de esto es el siguiente caso:

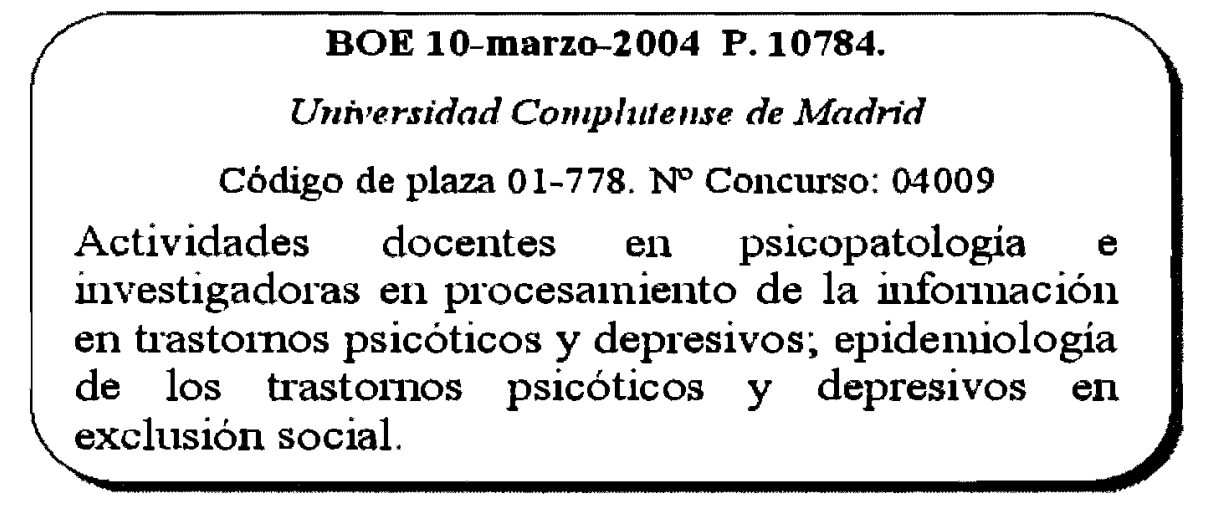

Es evidente que el anterior perfil es excesivamente especifico, en lo que respecta a la investigación, y el problema es que eso se valora como mérito. Pero en cualquier caso, carece de sentido, pues el candidato que gane el Concurso de acceso no tiene la obligación de investigar en esa línea una vez que se incorpore. En definitiva, el Real Decreto deja total libertad a las universidades para reglamentar los concursos de acceso, y eso ha llevado a que hay casi tantos formatos distintos en el Concurso de acceso como universidades, asi varía entre algunos muy complejos en los que se trata de una oposición hasta otros más simples en los que tan sólo hay que enviar el curriculum y una comisión lo evalúa sin la presencia del candidato. El hecho es que de una u otra forma se potencia que gane el Concurso "el candidato de casa", recuperando así algo que afortunadamente se había perdido con la derogación de la LRU. Si lo habitual es que los miembros de las comisiones de los concursos de acceso y los perfiles son propuestos por cada universidad y a 
su vez estas consultan a los departamentos, pues la lógica del "efecto domino" hace que los miembros de los departamentos traten de favorecer a los candidatos de casa, pues además de la "solidaridad", se pone en peligro la reconversión de los profesores contratados, máxime en los casos de Cátedra de Universidad, pues si gana el Concurso de acceso "el candidato de fuera" "el candidato de casa" continua con su plaza de funcionario y por tanto se dota una plaza nueva a ese departamento, para la cual no hay docencia, y ante esto sólo hay dos opciones, o que la universidad pague un salario adicional de un profesor que no necesita o bien que no se renueve el contrato a uno de los profesores no funcionarios. Por tanto, se reproduce el viejo vicio de la LRU que consiste en que la plaza debe ser para el candidato de la casa.

\section{DISCUSIÓN}

Lo primero que hay que dejar claro es que el detallado análisis crítico del Sistema Nacional de Habilitación y del Concurso de acceso no debe interpretarse como que si se tratase de un sistema caótico de selección de profesorado, pues ni eso es lo que se pretende, ni esa es la realidad en el marco de la historia de la selección del profesorado funcionario universitario en España. De hecho, si se compara la Habilitación Nacional con la LRU que le precede, no hay ringuna duda de que se trata de un sistema considerablemente mejor, y ello especialmente en su estructura más básica. Sólo como ejemplo, comparar una Comisión de evaluación con cinco miembros de los cuales dos son propuestos por la Universidad que convoca la plaza, frente a una Comisión con siete miembros con mayor cualificación (con un mínimo de tramos de investigación) y todos designados por sorteo, ya estamos partiendo de unos "cimientos más seguros". Por tanto, quede dicho que no es la intención tratar de invalidar el sistema actual de selección de profesorado universitario funcionario, sino que la intención es realizar un pormenorizado análisis para tratar de ver las pegas más importantes, que se ponen de manifiesto después de dos años de puesta en práctica, y como éstas pueden subsanarse para mejorar el sistema de selección. 
El análisis del Sistema de Habilitación pone de manifiesto que los criterios de evaluación son tan pocos y tan ambiguos que es casi es lo mismo que decir que no hay criterios y que aunque estos pueden ser definidos por la Comisión de evaluación, en la mayoría de los casos, al menos en Psicología, tampoco son definidos, al menos de una forma operativa. Lo mismo ocurre con el baremo y con el proceso de evaluación. Esto genera importantes problemas de fiabilidad y validez en la selección de profesores, tal como se ha puesto de manifiesto con algunos ejemplos de casos reales mencionados en este artículo.

Las recomendaciones que aquí se hacen de forma explícita o implicita sobre los criterios, baremos y proceso de evaluación pueden encontrar desde grandes defensores hasta grandes detractores. Asi, habria algunos que defenderian que el único sistema válido y fiable para seleccionar profesores es aquel que tiene definido de forma clara los criterios, el baremo y el procedimiento de evaluación, y en el otro extremo habría otros que hasta podrian denominar a un sistema rígido como una "cuanticofrenia". Pero lo cierto es que la ausencia o la ambigūedad de criterios permite actuaciones de comisiones de evaluación como las que se han comentado, el caso de un candidato que no pasa la primera prueba de una Habilitación a Cátedra de Universidad y meses después es habilitado por otra comisión. Y se podrían poner otros muchos ejemplos, como el caso de una profesora que consigue un tramo de investigación con ninguna publicación y con ningún congreso; o el caso de un profesor titular que después de 32 años trabajando como profesor en una universidad y que con una producción cientifica que se sintetiza en: ninguna tesis dirigida, ningún proyecto de investigación como investigador principal, ningún libro en editoriales científicas, y tan sólo diecisiete artículos en revistas científicas nacionales (de los cuales sólo dos son empíricos); y con estos "méritos" consigue que le nombren "profesor emérito"; pero lo más increíble del caso es que no sólo fue propuesto por unanimidad por todos los miembros de un departamento, sino que además pasó por varías comisiones de evaluación hasta conseguir con todos "esos méritos" más el "mérito de esperar que pasen los años" el nombramiento de profesor emérito, aunque quizás seria más justo decir "profesor deménito". Sin duda, 
este es un buen ejemplo de como se puede poner un nivel tan bajo para asegurarse de que cualquiera que lo solicite, lo consigue. Es muy probable que en España, en la historia de la selección de profesores, en los últimos años, hay muchos casos como estos, y ello son buenas demostraciones de lo que puede ocurrir cuando los miembros de las comisiones de evaluación actúan con total libertad de criterios y baremos.

Es evidente, y supuestamente compartido por todos que no existe un único modelo de "un buen profesor universitario", de hecho, el estudio de Agudelo, Bretón-López, Poveda-Vera et al. (2003) pone de manifiesto cómo distintos estilos de trabajo en la dirección de tesis doctorales pueden ser iguales de exitosos, pero en cualquier caso, el éxito se mide por la producción en tesis doctorales. En otras palabras, si se mide productividad científica no se está forzando a que todos trabajen de la misma forma y en los mismos temas. Aunque también es cierto, como dice Pelechano (2004), que la protocolización y automatismos en la evaluación y selección de profesores no parecen ir de la mano de la creatividad científica, dado que la acumulación de datos y resultados no genera creación sino publicaciones meritorias. Pero esto debe entenderse en su justa medida, pues este mismo autor sostiene que la creatividad y la genialidad en ciencia no es un rasgo permanente ni de puesta en acción diaria (Pelechano, 2005a) y además es algo característico de muy pocos investigadores e incluso de un periodo de la vida del investigador (Kanazawa, 2003). Por ello, aunque se asuma que en los sistemas más rígidos y estructurados de evaluación de profesores pueden, en alguna medida, no valorar la creatividad científica, la realidad es que esto afecta a pocos casos, y por otra parte, no se ha demostrado que sistemas de selección sin criterios definidos previamente tengan una mayor capacidad para evaluar la creatividad científica.

En función de lo anteriormente expuesto se concluye que el actual Sistema Nacional de Habilitación y el Concurso de Acceso constituyen un buen sistema de selección del profesorado funcionario universitario, siempre y cuando se mejoren los aspectos mencionados. Asi, en cuanto a los criterios, deben establecerse previamente y definirse de forma clara, por ejemplo, si los artículos se deben 
evaluar en función de donde se publican sigue siendo objeto de una gran polémica (véase, Buela-Casal, 2002, 2003; Garfield, 2003; Gil Roales-Nieto y Luciano, 2002; Pelechano, 2002a, 2002b, 2202c; Sternberg, 2003). Además, ya se ha demostrado que las revistas que no están incluidas en el Journal Citation Report también tienen factor de impacto y que este puede ser calculado exactamente de la misma forma que lo hace el Institute for Scientific Information (Buela-Casal, Carretero-Dios y Dantos-Roig, 2002; Buela-Casal et. al, 2004; Buela-Casal y López, 2005;) y un magnífico ejemplo es el reciente Índice de Impacto de Revistas Españolas de Ciencias Sociales (IN-RECS, 2005) que permite conocer el factor de impacto nacional e internacional de las revistas científicas españolas. De igual forma ocurre con la dirección de tesis doctorales, que no está claramente establecido su valor como un mérito de investigación (Polaino, 2002), y con el proyecto docente, pues no está determinado si es mejor un formato clásico o uno más moderno como por ejemplo, basado en el modelo de competencias (Gutiérrez, 2005).

En definitiva, para mejorar el Sistema de Habilitación Nacional es necesario definir los criterios, el baremo y regular el proceso de evaluación, pues esta es la única manera de incrementar la validez y fiabilidad de un sistema de selección del profesorado. Esto debe ir en paralelo con una mejor selección de los miembros que forman parte de las comisiones de evaluación. Mejorar el sistema de evaluación y selección del profesorado funcionario no sólo es una cuestión que afecte a la productividad científica de las universidad, sino que también tiene importantes implicaciones éticas, pues como muy acertadamente resaltó Echeburúa (2002) "No hay nada más discriminatorio que la no discriminación por el esfuerzo y el mérito" (p.394).

\section{BIBLIOGRAFÍA}

Agudelo, D., Bretón-López, J., Ortiz-Recio, G., Poveda-Vera, J., Teva, I., Valor-Segura, I. y Vico, C. (2003). Análisis de la productividad científica de la Psicología española a través de las tesis doctorales. Psicothema, 15, 595-609. 
Agudelo, D., Bretón-López, J., Poveda-Vera, J., Teva, I., Valor-Segura, I. y Vico, C. (2003). ¿Cómo tener éxito en un doctorado en Psicología? Opinión de los directores de tesis doctorales más productivos de España. International Journal of Clinical and Health Psychology, 3, 565-593.

Buela-Casal, G. (2001). La psicología española y su proyección intemacional. El problema del criterio: internacional, calidad y castellano y/o inglés. Papeles del Psicologo, 21, 53-57.

Buela-Casal, G. (2002). La evaluación de la investigación científica: el criterio de la opinión de la mayoría, el factor de impacto, el factor de prestigioy "Los Diez Mandamientos" para incrementar las citas. Análisis y Modificación de Conducta, 28, 455-475.

Buela-Casal, G. (2003). Evaluación de la calidad de los artículos y de las revistas científicas: propuesta del factor de impacto ponderado y de un indice de calidad. Psicothema, 15, 23-35.

Buela-Casal, G. (2005). Situación actual de la productividad científica de las universidades españolas. Intemational Joumal of Clinical and Health Psychology, 5, 175-190.

Buela-Casal, G., Carretero-Dios, H., De los Santos-Roig, M. (2002). Análisis comparativo de las revistas de Psicología en castellano con factor de impacto. Psicothema, 14, 837-852.

Buela-Casal, G., Fernández-Hermlda, J. R. y López, W. (2003). Iberindex: Índice Iberoamericano de evaluación de publicaciones científicas. Papeles del Psicologo, 23 (supl. Infocop), 41-44.

Buela-Casal, G., Medlna, A., Viedma, M.I., Godoy, V., Lozano, S. y Torres, G. (2004). Factor de impacto de tres revistas españolas de Psicología. Psicothema, 16, 681-689.

Buela-Casal, G. y López, W. (2005). Evaluación de las revistas científicas iberoamericanas de Psicologia. Iniciativas y estado actual. Revista Latinoamericana de Psicología, 37, (en prensa).

Buela-Casal, G., Perakakls, P., Taylor, M. y Checa, P. (2005). Measuring Intemationality: Reflections and perspectives on academic journals. Manuscrito enviado para publicación.

Echeburúa, E. (2002). El sistema de sexenios en la evaluación de la actividad investigadora en el ámbito de la psicología clínica: Una primera reflexión. Análisis y Modificación de Conducta, 28, 391-404.

Garfield, E. (2003). The meaning of the impact factor. Intemational Journal of Clinical and Health Psychology, 3, 363-369.

GIl Roales-Nleto, J. y Luciano, M. C. (2002). A la calidad por the quantity (porque la cantidad no vale). Algunas reflexiones sobre los criterios de evaluación de la evaluación de la calidad de la investigación psicológica. Análisis y Modificación de Conducta, 28, 431-454 
Gordillo, V., Gonzáles-Marqués, J. y Muñlz, J. (2004). La evaluación de proyectos de investigación por la ANEP. Psicothema, 16, 343-349.

Gutierrez, 0. (2005). Educación y entrenamiento basados en el concepto de competencia: implicaciones para la acreditación de los programas de Psicología. . Revista Mexicana de Psicología, 22, (en prensa).

IN-RECS (2005). Indice de Impacto de Revistas Españolas de Ciencias Sociales.http://ec3.ugr.es/in-recs/index2.shtml

Montero, I. y León, O. (2005). Sistemas de Clasificación del método en los informes de investigación en Psicologia. Intemational Joumal of Clinical and Health Psychology, 5, 115-127.

Kanazawa, S. (2003). Why productivity fades with age: The crime-genius connection. Joumal of Research Personality, 37, 257-272.

Pelechano, V. (2000). Psicología sistemática de la personalidad. Barcelona: Ariel

Pelechano, V. (2002a). Epílogo del Monográfico 2002: La valoración de la actividad científica. Análisis y Modificación de Conducta, 28, 477-479.

Pelechano, V. (2002b). Presentación del Monográfico 2002: La valoración de la actividad científica. Análisis y Modificación de Conducta, 28, 311316.

Pelechano, V. (2002c). ¿Valoración de la actividad científica en psicologia? ¿Pseudoproblema, sociologismo o ideologismo? Análisis y Modificación de Conducta, 28, 323-362.

Pelechano, V. (2004, septiembre). Comunicación, difusión y calidad: el papel de la normatividad y la diversidad en la creatividad científica. Conferencia presentada en el Curso sobre Evaluación y Acreditación de la Calidad de la Educación Superior. ANECA-UGR.Almuñecar. http:// www.aneca.es/actividades/eventos/activ_granada2004.html

Pelechano, V. (2005a). La valoración de programas de doctorado en Psicología: Una cuestión abierta. Revista Mexicana de Psicologia, 22, (en prensa).

Pelechano, V. (2005b). Marco general con una propuesta de contenidos mínimos en Psicología: Una cuestión abierta. Revista Mexicana de Psicología, 22, (en prensa).

Pérez, M. (2002). La psicología en el contexto de la cultura española. Análisis y Modificación de Conducta, 28, 405-430.

Polaino, A. (2002). Ciencia, política y política de la investigación. Análisis y Modificación de Conducta, 28, 363-390

Raina, K.C. y Mahoney, M. (2005). Los orígenes del doctorado en Psicología. En G. Buela-Casal (Dir.), Manual práctico para realizar un doctorado en Psicología y Ciencias de la Educación (en prensa). 
Ramos-Alvarez, M.M. y Catena, A. (2004). Normas para la elaboración y revisión de artículos originales experimentales en ciencias del comportamiento. International Joumal of Clinical and Health Psychology, 4, 173189.

Sternberg, R. J. (2003). There is no place for hostile reviews. International Journal of Clinical and Health Psychology, 3, 159-161. 